OnLine Journal of Biological Sciences 7 (1): 44-51, 2007

ISSN 1608-4217

(C) 2007 Science Publications

\title{
Characterization of Bacterial Strains Isolated Through Microbial Profiling of Urine Samples
}

\author{
Poulomi Nandy, A.R. Thakur and S. Ray Chaudhuri \\ Department of Biotechnology, West Bengal University of Technology, \\ BF-142, Sector 1, Salt lake, 700064, Kolkata, India
}

\begin{abstract}
The present study was conducted to determine the microbial profile in urine samples. Differential and selective chromogenic culture media were used for the rapid detection, identification and enumeration of urinary tract pathogens namely, E. coli, Pseudomonas aeruginosa, Klebsiella pneumoniae, Staphylococcus aureus, Enterococcus faecalis, Proteus mirabilis. Urine samples of normal healthy individuals as well as patients with Urinary Tract Infection (UTI) were screened on hicrome agar plates. The cultivable bacteria present in urine were isolated based on chromogenic detection. Antibiotic sensitivity assay, morphological characterization and biochemical tests, namely protease, oxidase, catalase, lipase, DNase and lecithinase assay were performed with the 15 isolates obtained from urine samples. The molecular analyses of the isolates were done through partial sequencing of the 16SrDNA gene; six of them were found to be novel and submitted in GenBank under the accession numbers EF644491-96. Phylogenetic tree of the isolates were constructed by neighbour joining method.
\end{abstract}

Key words: Urinary tract microorganisms, chromogenic media, phylogenetic analysis, biochemical characterization

\section{INTRODUCTION}

Urinary Tract Infection, commonly known as UTI, affects as many as $50 \%$ women at least once during their lifetime and $25 \%$ of those who acquire UTI, will have recurrent infection within the following six months ${ }^{[1]}$. Urine located within the urinary tract, excluding the distal region of the urethra is considered sterile in healthy individuals, as indicated by the absence of cultivable bacterial cells. Urinary Tract Infection (UTI) describes a condition in which there are micro-organisms established and multiplying within the urinary tract. It is most often due to bacteria (95\%), but may also include fungal and viral infection ${ }^{[2]}$. In general UTI is characterized by the presence of bacteria in bladder urine. Uropathogenic E. coli is responsible for approximately $85 \%$ of community acquired infections, besides Proteus, Klebsiella and Pseudomonas ${ }^{[3,4,5,6]}$. On the basis of the work done by Kass, $10^{5}$ Colony Forming Units (CFU) of a single species per $\mathrm{ml}$ in a clean catch midstream sample of urine is considered as significant bacteriuria ${ }^{[7,8]}$. While this threshold still holds in asymptomatic patients, in many circumstances a lower threshold is considered significant for symptomatic patients ${ }^{[9]}$.
Chromogenic substrates have been widely used in culture media for the detection, isolation, identification and enumeration of micro organisms in clinical samples. Chromogenic media incorporates enzymatic methods using specific chromogenic substrates added to primary selective and nonselective media. The chromogenic mixture contains chromogenic substrates as Salmon-GAL, X-GAL, X-glucoronide. Colourless substrates produce characteristic colours when cleaved by organism-specific enzymes ${ }^{[10]}$, for example, formation of blue coloured colonies due to cleavage of the chromogenic substrate by beta-glucosidase, possessed by Enterococci. E. coli produces pink colonies due to the presence of the enzyme beta-Dgalactosidase which cleaves the chromogenic substrate. Presence of rich source of phenylalanine and tryptophan from peptone and tryptone in the media provides an indication of tryptophan deaminase activity indicating the presence of Proteus $s p$. which appears brown $^{[11]}$.

Moreover, even in the absence of detectable urinary tract malformations, making way for infections, many patients, have repeated episodes of UTI, which are often asymptomatic. UTI in pregnancy maybe associated with an increase in neonatal mortality and it

Corresponding Author: Shaon Ray Chaudhuri, Department of Biotechnology, West Bengal University of Technology, BF-142, Sector 1, Salt lake, 700064, Kolkata, India, Tel: +91 3323210731 
can also be a source for Gram negative septicemia, which so frequently proves fatal ${ }^{[12,13]}$. Thus these infections leave their mark from cradle to the grave and are responsible for many complications. Hence it is important to diagnose and treat UTI before it produces symptoms, since this would offer the prospect of reducing morbidity and decrease the work load of costly dialysis and transplant units.

The emperical choice of an effective treatment is becoming more difficult as urinary pathogens are increasingly becoming resistant to commonly used antibiotics $^{[14,15,16]}$. The degree to which a causative organism requires identification varies, but identification is most useful in complex clinical cases, for e.g. to distinguish relapse, to indicate failure of an antibiotic treatment and to detect reinfection with a different organism in patients with recurrent infections ${ }^{[2]}$.

Therefore, the objective of this study was to identify the spectrum of bacteria inhabiting the urinary tract among women population in and around Kolkata, India. This microbial detection was done by the culture dependent method, which is considered to be the golden standard for urine sample analysis. Once the microbes were identified, their antibiotic sensitivity profiles were studied, which can be used as an important tool in the treatment of the infection and would also produce a clear picture of the resistance acquired by the microbes to the commonly used antibiotics. The phylogenetic analysis of the isolates, especially the novel ones were important for revealing the identity of the organism.

\section{MATERIALS AND METHODS}

Chromogenic media used for microbial isolation: Four different types of chromogenic agar plates by Himedia was used for screening the urine samples - Hi Crome UTI Agar (M1353, LOT WF256); Hi Crome Kleb Selective Agar Base (M1573, LOTWC154), Klebsiella Selective Supplement (FD225); Pseudomonas Agar (M120, LOTWG213); Hi Crome Aureus Agar Base (M1468, LOTWE080) and Egg Yolk Tellurite Emulsion (FD046). The media was prepared as per the manufacturer's protocol and sterilized by autoclaving at $15 \mathrm{psi}$ for $15 \mathrm{~min}$.

Isolation of UTI causing microbes: Samples were regularly procured from Pathology Department of Peerless Nursing Home, Kolkata and N. R. S. Medical College and Hospital, Kolkata. A survey with sample size of 200 of the local women population which includes normal healthy individuals as well as infected hospitalized subjects was done. Early morning midstream urine samples were collected in sterile $15 \mathrm{ml}$ falcon tubes (Tarson). 50 $\mu 1$ of urine sample from each of the subjects was spread on the four different types of hicrome agar plates (UTI, Pseudomonas, Klebsiella, Aureus plates). The plates were incubated overnight at $37^{\circ} \mathrm{C}$. Next day the number of colonies (differentially coloured) were counted and the Colony Forming Units per milliliter $(\mathrm{CFU} / \mathrm{ml})$ determined. Pure, isolated colonies of each type was separately cultured and characterized.

Morphological characterization: The colony character was noted and the Gram nature of the fifteen isolates were determined by observing in bright field the Gram stained slides of the isolates under $40 \mathrm{X}$ magnification of Axiostar Plus fluorescence microscope from Zeiss.

\section{Biochemical Characterization}

Protease assay: Milk media containing double toned milk (10\%), yeast extract $(0.3 \%)$ and Agar (1.5\%) was sterilized at $15 \mathrm{psi}$ for 15 minutes. The isolates were streaked on milk media plates and left overnight at their respective optimum temperature. The plates were observed for growth and production of clearing zone around the growth.

Oxidase test: Oxidase discs from Himedia (DD018) were used to test the presence of the enzyme cytochrome oxidase. A loopful of bacterial cell from solid media was brought in contact with the oxidase disc and the change in colour was observed within 60 seconds.

Catalase test: Catalase assay was done by applying 3\% Hydrogen peroxide (Merck India) directly over the colonies on the solid media.

Lipase test: Tributyrin agar (Himedia M157) with tributyrin oil (Himedia FD08) added as a supplement was the differential medium used for the detection of lipase activity. The isolates were streaked on these plates and left overnight at their respective optimum temperature. The plates were observed for growth and production of clearing zone around the growth.

DNase test: DNase agar (Himedia M482) with Toluidine blue (Himedia FD05) added as a supplement was used for the detection of the enzyme DNase. The isolates were streaked on these plates and left overnight at their respective optimum temperature. The plates were observed for growth and the appearance of bright zone in the region of growth. 
OnLine J. Biol. Sci., 7 (1): 44-51, 2007

Table 1: Morphological characterization of the bacterial isolates as observed by gram staining under axiostar plus fluorescence microscope from zeiss at 100X magnification

\begin{tabular}{llll}
\hline Strain & Appearance of the colony & Cell morphology & Gram nature \\
\hline A & $1 \mathrm{~mm}$, entire, white, opaque & Coccus & Gram Positive \\
B & $<1 \mathrm{~mm}$, entire, colourless, translucent & Bacillus & Gram Positive \\
C & $1 \mathrm{~mm}$, entire, white, translucent & Bacillus & Gram Negative \\
D & $1.5 \mathrm{~mm}$, entire, golden yellow, opaque & Coccus & Gram Positive \\
E & $2 \mathrm{~mm}$, slimy, greenish yellow, translucent & Bacillus & Gram Negative \\
F & $2 \mathrm{~mm}$, entire, colourless, translucent & Bacillus & Gram Negative \\
G & $2 \mathrm{~mm}$, entire, colourless, translucent & Coccus & Gram Negative \\
H & $2 \mathrm{~mm}$, entire, white, opaque & Bacillus & Gram Negative \\
I & $1 \mathrm{~mm}$, entire, white, opaque & Coccus & Gram Positive \\
J & $2 \mathrm{~mm}$, entire, white, opaque & Coccus & Gram Positive \\
K & $2 \mathrm{~mm}$, entire, colourless, translucent & Bacillus & Gram Negative \\
L & $1 \mathrm{~mm}$, entire, cream, translucent & Coccus & Gram Positive \\
M & $2.5 \mathrm{~mm}$, entire, white, opaque & Bacillus & Gram Negative \\
N & $2 \mathrm{~mm}$, entire, creamish yellow, translucent & Coccus & Gram Positive \\
O & $1.5 \mathrm{~mm}$, entire, white, translucent & Bacillus & Gram Negative \\
\hline
\end{tabular}

Lecithinase test: The isolates were also streaked on Hicrome Aureus Agar (Himedia M1468) and Egg Yolk Tellurite Emulsion (Himedia FD046) added as a supplement to detect the presence of enzyme lecithinase.

Antibiotic assay: The sensitivity of the strains against various antibiotics was determined by using antibiotic sensitivity discs; namely Ampicillin (A), Cephadroxil (Cq), Chloramphenicol (C), Cloxacillin (Cx), Cephotaxime $(\mathrm{Ce})$, Ceftazidime $(\mathrm{Ca})$, Ciprofloxacin (Cf), Doxycycline Hydrochloride (Do), Gentamicin (G), Metronidazole (Mt), Neomycin (N), Norfloxacin (Nx), Polymyxin B (Pb), Rifampicin (R), Roxithromycin (Ro), Tetracycline (T), Trimethoprin (Tr), Vancomycin (Va). We analyzed the antibiotic resistant characteristics of the 15 isolates by disk diffusion method. The assay was conducted in triplicate for each organism evaluated. Mueller Hinton Agar (MHA) by Himedia (M173), were used to evaluate each of the microorganisms for antibiotic resistance. A 100 times diluted log phase culture of each isolate was poured on MHA plates.

It was swirled to uniformly distribute the culture throughout the plate. After waiting for about half an hour, the cultures were carefully pipette out from the plates and the plates were allowed to dry before the disks containing the antibiotics were placed onto the MHA surface using the dispenser provided with the antibiotic kit. The disks were allowed to settle before being inverted and placed in an incubator at $37^{\circ} \mathrm{C}$. The plates were checked for susceptibility after 24 hours. The zones of inhibition were recorded for all the plates and a determination was made as to whether the microorganisms were susceptible, intermediately resistant or resistant to each antibiotic evaluated using National Committee for Clinical Laboratory Standard's (NCCLS) chart provided with the antibiotic kits by Himedia.
Table 2: Reflecting the results of biochemical characterization of the fifteen different bacterial isolates

\begin{tabular}{|c|c|c|c|c|c|c|}
\hline Strains & Protease & Oxidase & Catalase & Lipase & DNase & Lecithinase \\
\hline $\mathrm{A}$ & - & - & + & - & - & + \\
\hline B & - & - & - & - & - & - \\
\hline C & - & + & - & - & - & - \\
\hline $\mathrm{D}$ & - & - & + & - & - & + \\
\hline E & + & + & + & - & + & - \\
\hline $\mathrm{F}$ & - & - & + & - & - & - \\
\hline G & - & + & - & - & + & - \\
\hline $\mathrm{H}$ & - & - & - & - & - & - \\
\hline I & - & - & + & - & - & - \\
\hline $\mathrm{J}$ & - & - & + & - & - & - \\
\hline $\mathrm{K}$ & - & - & - & - & - & - \\
\hline $\mathrm{L}$ & - & - & + & - & - & + \\
\hline $\mathrm{M}$ & - & - & + & - & - & - \\
\hline $\mathrm{N}$ & + & - & + & - & - & - \\
\hline $\mathrm{O}$ & - & - & - & - & - & - \\
\hline
\end{tabular}

Note: Presence of the respective enzymatic property(+) i.e. showing a positive biochemical reaction; absence of the enzymatic property $(-)$

Molecular characterization: DNA of the 15 isolates was extracted from overnight grown culture by using PrepMan Ultra Kit from ABI according to manufacturer's protocol and sent for sequencing. The sequences were subjected to blast analysis. The novel sequences were submitted to GenBank. The sequences were also subjected to phylogenetic analysis using neighbour joining method.

\section{RESULTS AND DISCUSSION}

Eight out of the fifteen isolates were found to be Gram negative. Their morphological characterization is cited in Table 1. The biochemical characterization in Table 2 reveals nine strains were catalase positive. This indicates that these microbes possess the defence mechanism against reactive oxygen species and oxidative stress and thereby help them to survive in an aerobic environment. 
OnLine J. Biol. Sci., 7 (1): 44-51, 2007

Table 3: Molecular characterization of the isolates as depicted through their phylogenetic analysis

\begin{tabular}{llcc}
\hline Strain & Maximum similarity with organism & Percentage similarity & Accession number \\
\hline A & Staphylococcus haemolyticus & 99.89 & EF644492 \\
B & Bacillus pumilus & 99.14 & - \\
C & Klebsiella pneumoniae & 99.6 & EF6444491 \\
D & Staphylococcus aureus & 100 & - \\
E & Pseudomonas aeruginosa & 100 & - \\
F & Escherichia coli O157 H7 & 100 & - \\
G & Aeromonas caviae & 100 & - \\
H & Klebsiella pneumoniae & 99.6 & EF6444493 \\
I & Staphylococcus haemolyticus & 99.72 & EF644494 \\
J & Staphylococcus haemolyticus & 99.58 & EF644495 \\
K & Escherichia coli O157 H7 & 100 & - \\
L & Staphylococcus aureus & 100 & - \\
M & Klebsiella pneumoniae & 99.62 & - \\
N & Staphylococcus sciuri & 100 & - \\
O & Klebsiella pneumoniae & 99.57 & EF6444966 \\
\hline
\end{tabular}

Table 4: Results of antibiotic sensitivity of the fifteen isolates using Muller Hinton Agar. The abbreviation of the antibiotics are Ampicillin (A), Cephadroxil (Cq), Chloramphenicol (C), Cloxacillin (Cx), Cephotaxime (Ce), Ceftazidime (Ca), Ciprofloxacin (Cf), Doxycycline Hydrochloride (Do), Gentamicin (G), Metronidazole (Mt), Neomycin (N), Norfloxacin $(\mathrm{Nx})$, Polymyxin B $(\mathrm{Pb})$, Rifampicin $(\mathrm{R})$, Roxithromycin (Ro), Tetracycline (T), Trimethoprin (Tr) and Vancomycin (Va) with their respective concentration in meg per disc is mentioned in the first row of the table, while the first column denotes the different strains

\begin{tabular}{|c|c|c|c|c|c|c|c|c|c|c|c|c|c|c|c|c|c|c|}
\hline Strain & Т 30 & $\mathrm{Cq} 30$ & C 30 & Cx 10 & $\mathrm{~Pb} 100$ & N 30 & A 10 & R 15 & Cf 5 & Ro 30 & D 30 & Ce 30 & Ca 30 & Nx 10 & G 10 & Mt 4 & $\operatorname{Tr} 30$ & Va 30 \\
\hline$\overline{\mathrm{A}}$ & $\mathrm{S}$ & $S$ & $\mathrm{~S}$ & $\mathrm{~S}$ & $\mathrm{R}$ & $\mathrm{S}$ & I & $\mathrm{S}$ & $\mathrm{S}$ & I & $\mathrm{S}$ & I & I & $\mathrm{S}$ & $\mathrm{S}$ & $\mathrm{R}$ & $\mathrm{S}$ & $S$ \\
\hline B & $\mathrm{R}$ & I & $\mathrm{R}$ & $\mathrm{R}$ & $\mathrm{R}$ & $\mathrm{R}$ & S & S & $\mathrm{R}$ & $\mathrm{R}$ & $\mathrm{R}$ & $\mathrm{R}$ & S & $\mathrm{R}$ & $\mathrm{R}$ & $\mathrm{R}$ & S & S \\
\hline C & $\mathrm{R}$ & I & $\mathrm{R}$ & $\mathrm{R}$ & $\mathrm{R}$ & I & $S$ & $S$ & $\mathrm{R}$ & $\mathrm{R}$ & $\mathrm{R}$ & $\mathrm{S}$ & $\mathrm{R}$ & $\mathrm{R}$ & $\mathrm{R}$ & $\mathrm{R}$ & $\mathrm{S}$ & $S$ \\
\hline D & S & S & S & S & $\mathrm{R}$ & S & $\mathrm{S}$ & S & $\mathrm{R}$ & S & S & S & S & $\mathrm{R}$ & S & $\mathrm{R}$ & S & S \\
\hline E & $\mathrm{R}$ & $\mathrm{R}$ & $\mathrm{R}$ & $\mathrm{R}$ & $\mathrm{R}$ & $\mathrm{R}$ & $\mathrm{R}$ & $\mathrm{R}$ & $\mathrm{R}$ & $\mathrm{R}$ & $\mathrm{R}$ & I & $\mathrm{R}$ & $\mathrm{R}$ & $\mathrm{R}$ & $\mathrm{R}$ & $\mathrm{R}$ & $\mathrm{R}$ \\
\hline $\mathrm{F}$ & S & I & S & $\mathrm{R}$ & $\mathrm{R}$ & I & I & $\mathrm{R}$ & $\mathrm{R}$ & $\mathrm{R}$ & S & S & S & $\mathrm{R}$ & S & $\mathrm{R}$ & S & $\mathrm{R}$ \\
\hline G & I & $\mathrm{R}$ & $S$ & $\mathrm{R}$ & $\mathrm{R}$ & S & I & I & $\mathrm{R}$ & S & $\mathrm{R}$ & $\mathrm{R}$ & $\mathrm{R}$ & $\mathrm{R}$ & S & $\mathrm{R}$ & S & $\mathrm{R}$ \\
\hline $\mathrm{H}$ & S & S & S & S & $\mathrm{R}$ & S & S & S & S & S & S & S & $\mathrm{R}$ & S & S & $\mathrm{R}$ & S & $\mathrm{R}$ \\
\hline I & S & S & S & I & $\mathrm{R}$ & S & $\mathrm{R}$ & S & $S$ & S & S & S & $\mathrm{R}$ & S & S & $\mathrm{R}$ & S & S \\
\hline $\mathrm{J}$ & S & S & S & S & $\mathrm{R}$ & S & S & S & $\mathrm{R}$ & $\mathrm{R}$ & S & S & S & $\mathrm{R}$ & S & $\mathrm{R}$ & S & S \\
\hline K & $\mathrm{R}$ & I & S & $\mathrm{R}$ & $\mathrm{R}$ & $\mathrm{R}$ & $\mathrm{R}$ & $\mathrm{R}$ & $\mathrm{R}$ & $\mathrm{R}$ & $\mathrm{R}$ & S & S & $\mathrm{R}$ & $\mathrm{R}$ & $\mathrm{R}$ & $\mathrm{R}$ & $\mathrm{R}$ \\
\hline $\mathrm{L}$ & S & S & S & S & $\mathrm{R}$ & S & $\mathrm{R}$ & S & S & S & S & S & I & S & S & $\mathrm{R}$ & $\mathrm{R}$ & I \\
\hline M & I & I & S & $\mathrm{R}$ & $\mathrm{R}$ & I & $\mathrm{R}$ & $\mathrm{R}$ & I & I & I & I & S & S & S & $\mathrm{R}$ & S & $\mathrm{R}$ \\
\hline $\mathrm{N}$ & S & S & S & I & $\mathrm{R}$ & S & S & S & I & S & S & S & $\mathrm{R}$ & S & S & I & S & I \\
\hline $\mathrm{O}$ & $\mathrm{S}$ & S & S & S & $\mathrm{R}$ & S & $\mathrm{S}$ & S & S & S & S & S & $\mathrm{S}$ & S & S & $\mathrm{R}$ & S & S \\
\hline
\end{tabular}

Note: $\mathrm{R}=$ Resistant, $\mathrm{I}=$ Intermediate, $\mathrm{S}=$ Sensitive

Two strains were found to be protease positive. This property can be commercially utilized provided the strains are non-pathogenic. Three of the strains were found to show lecithinase activity in egg yolk media. Strain A was found to show a similarity of $99.89 \%$ with Staphylococcus haemolyticus while D and L showed $100 \%$ similarity with S. aureus. Inspite of showing $100 \%$ homology with $S$. aureus, the isolates varied in the biochemical properties already reported for $S$. aureus. This may be attributed to the fact that only partial 16S rDNA sequence analysis was done for each of the isolates. The region amplified does not show the variation. Since there are other characteristic differences at the functional level so it is expected that there would be variation in the other parts of 16SrDNA to explain the functional variation.

The GenBank Accession number of the isolate, its closest cultivable neighbour through partial 16SrDNA sequence analysis and the percentage similarity with the latter are reprsented in Table 3. Isolate $\mathrm{A}, \mathrm{C}, \mathrm{H}, \mathrm{I}, \mathrm{J}$ and $\mathrm{O}$ were found to be novel. Figure 1-15 represents the phylogenetic tree of the isolates constructed by neighbour joining method. The antibiotic sensitivity data of the isolates is represented in Table 4.

The fact that majority of the microbes are catalase positive, indicates that they possess the defence mechanism against reactive oxygen species and oxidative stress and thereby are able to survive in an aerobic environment. In the protease assay, the casein present in milk acts as a substrate for the enzyme. Protease positive strains breakdown the casein and produce a clear zone around it. This property can be commercially utilized provided the strains $\mathrm{E}$ and $\mathrm{N}$ are non-pathogenic. Moreover, the presence of this enzyme might indicate the probable pathogenic nature of the microbes enabling their defence mechanism. In the oxidase test, cytochrome oxidase is an enzyme found in some bacteria that transfers electrons to oxygen, the 
pecimen : Specimen1
Join: $1.5 \%$

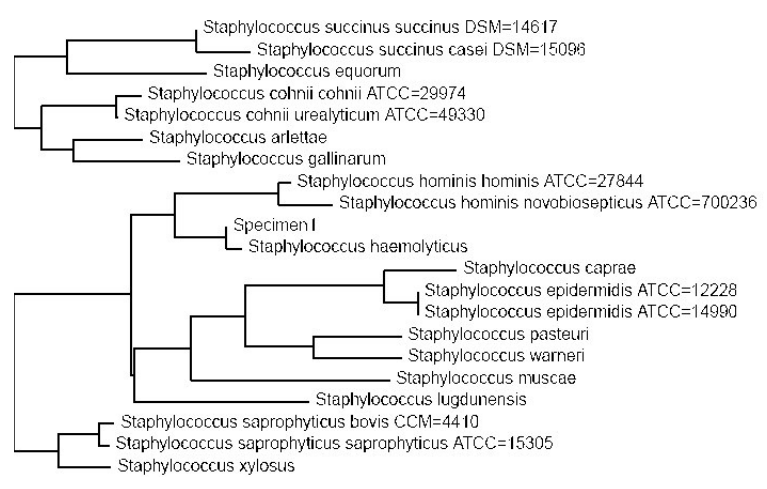

Fig. 1: Phylogenetic tree of Isolate A constructed using neighbour joining method, showing $99.89 \%$ similarity with Staphyloccus haemolyticus

Specinen: Specimen 4
N. Join: $3.5 \%$

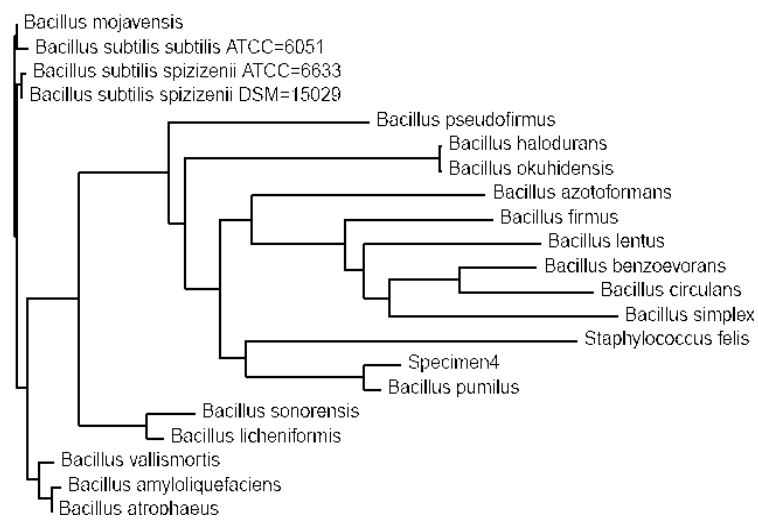

Fig. 2: Phylogenetic tree of Isolate B constructed using neighbour joining method, showing $99.14 \%$ similarity with Bacillus pumilus

final electron acceptor in some electron transport chains. If the bacteria oxidize the disk i.e. remove electrons from the disk, which acts as an electron donater to cytocrome oxidase, then it turns purple, indicating a positive test as shown by strains $\mathrm{C}, \mathrm{E}$ and G. Lipase breaks down lipid. Tributyrin oil is a type of triglyceride which forms an opaque suspension in agar. When an organism produces lipase and breaks down the tributyrin, a clear halo surrounds the area where the lipase producing organism has grown. None of the strains showed lipase production. DNase positive organisms $\mathrm{E}$ and $\mathrm{G}$ produce the enzyme which hydrolyzes DNA present in the medium. Toluidine blue present as an indicator forms a complex with

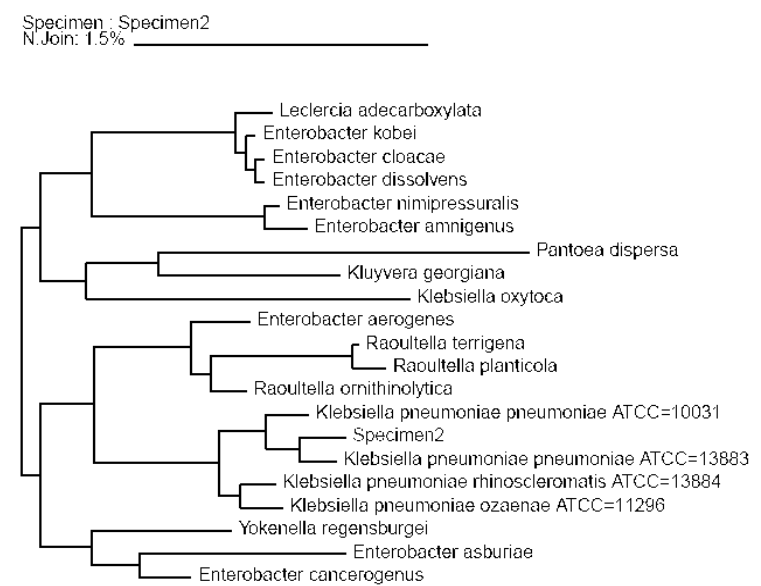

Fig. 3: Phylogenetic tree of Isolate $\mathrm{C}$ constructed using neighbour joining method, showing $99.6 \%$ similarity with $K$. pneumoniae

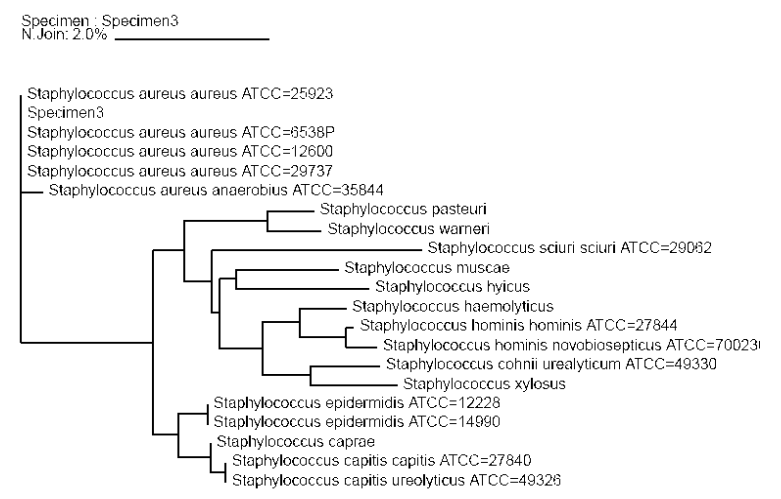

Fig. 4: Phylogenetic tree of Isolate D constructed using neighbour joining method, depicting 100\% homology with $S$. aureus

hydrolysed DNA to produce bright zones surrounding colonies on a royal blue background. Organisms positive for the enzyme lecithinase, strain $\mathrm{A}, \mathrm{D}$ and $\mathrm{L}$ produce typical black coloured colonies with a clear zone around it. Lecithinase is an enzyme known to be involved during invasion in pathogenesis.

The antibiotic susceptibility profile of the microbes reveal that they are becoming increasingly resistant to commonly used antibiotics, a fact which is of great concern to medical professionals. One interesting finding is that all the strains are resistant to antibiotic Polymyxin B and Metronidazole accept one. On the other hand majority of them are found to be sensitive to the antibiotic Chloramphenicol, which therefore can be used as a potent antimicrobial agent in the treatment of the infection. 
Specinen: Specimen2
N.Join: $30 \% \%$ p

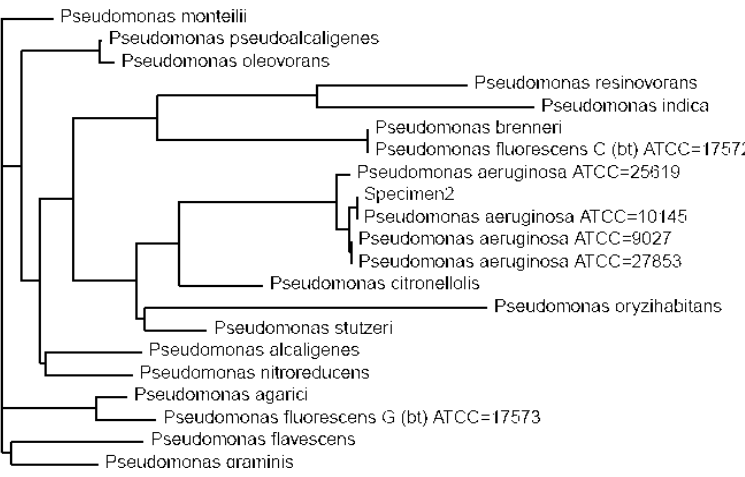

Fig. 5: Phylogenetic tree of Isolate E constructed using neighbour joining method, depicting 100\% homology with Pseudomonas aeruginosa

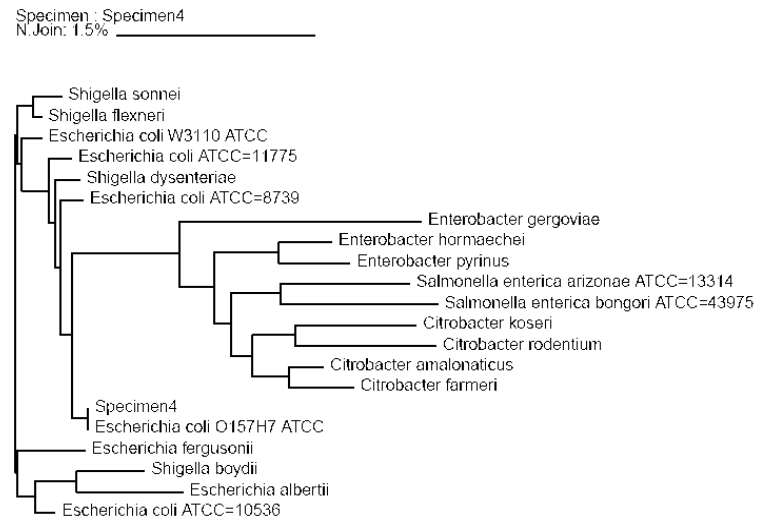

Fig. 6: Phylogenetic tree of Isolate F constructed using neighbour joining method,constructed using neighbour joining method, depicting 100\% homology with E. coli $0157 \mathrm{H} 7$

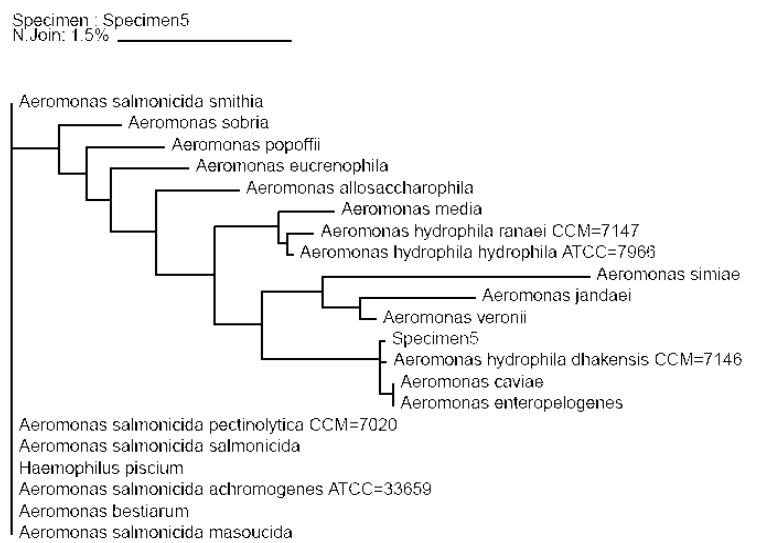

Fig. 7: Phylogenetic tree of Isolate G constructed using neighbour joining method, depicting 100\% homology with Aeromonas caviae
Specimen: Speciments

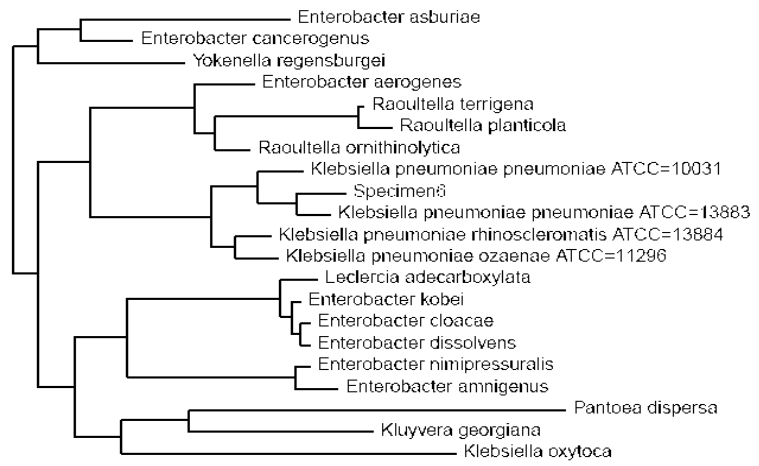

Fig. 8: Phylogenetic tree of Isolate $\mathrm{H}$ constructed using neighbour joining method, showing $99.6 \%$ similarity with $K$. pneumoniae

Specimen: Specimen 7
N. Join: $1.5 \%$,

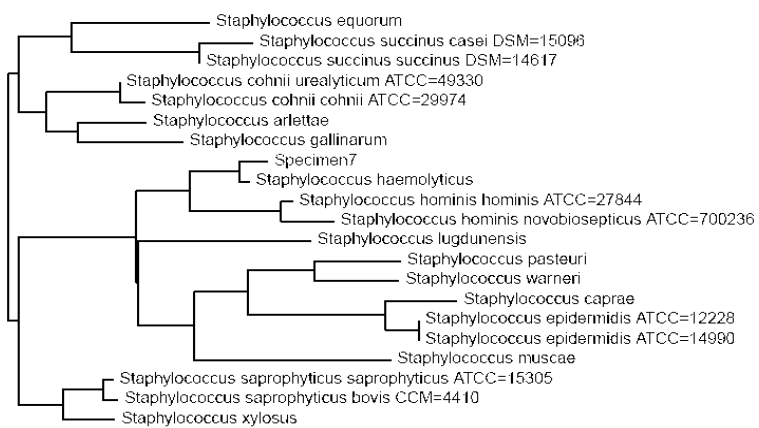

Fig. 9: Phylogenetic tree of Isolate I constructed using neighbour joining method, showing $99.72 \%$ similarity with $S$. haemolyticus

Specinen: Specimen 1
N. Join: $1.5 \%$.

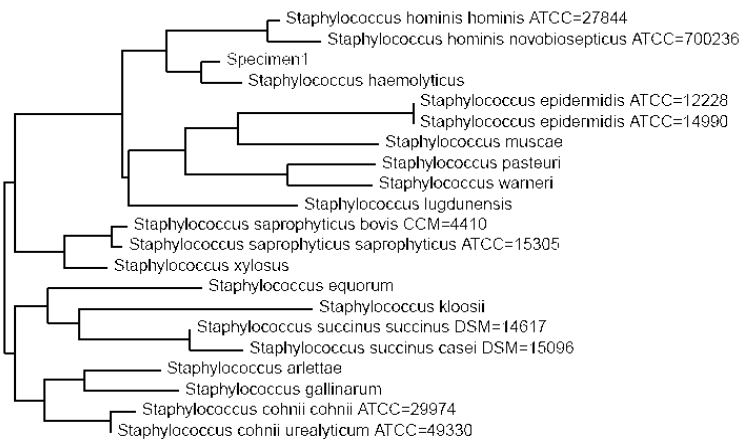

Fig. 10: Phylogenetic tree of Isolate $\mathrm{J}$ constructed using neighbour joining method,constructed using neighbour joining method, showing $99.58 \%$ similarity with $S$. haemolyticus 
OnLine J. Biol. Sci., 7 (1): 44-51, 2007

Specinen: Specimen-1
N. Join: $1.5 \% \%$

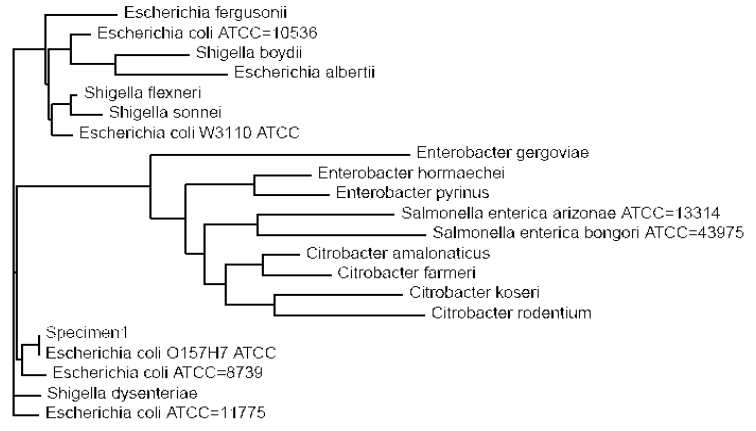

Fig. 11: Phylogenetic tree of Isolate $\mathrm{K}$ constructed using neighbour joining method, depicting $100 \%$ homology with E. coli $0157 \mathrm{H} 7$

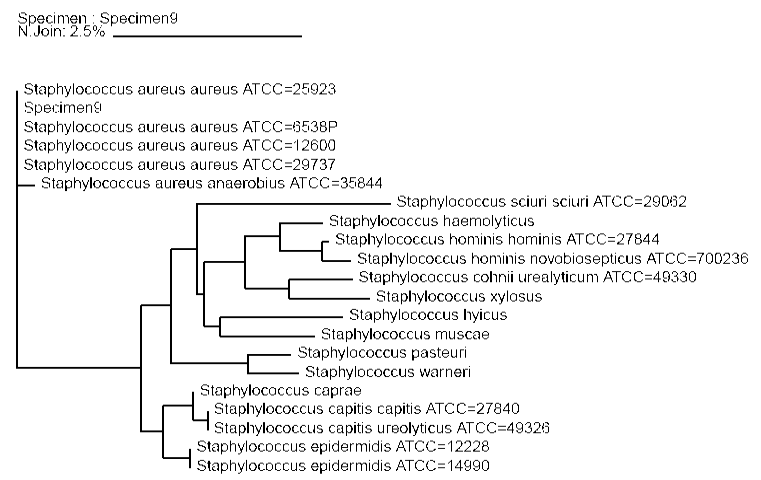

Fig. 12: Phylogenetic tree of Isolate L constructed using neighbour joining method, depicting $100 \%$ homology with S. aureus

Specinen: Specimen 10
N.Join: $15 \%$

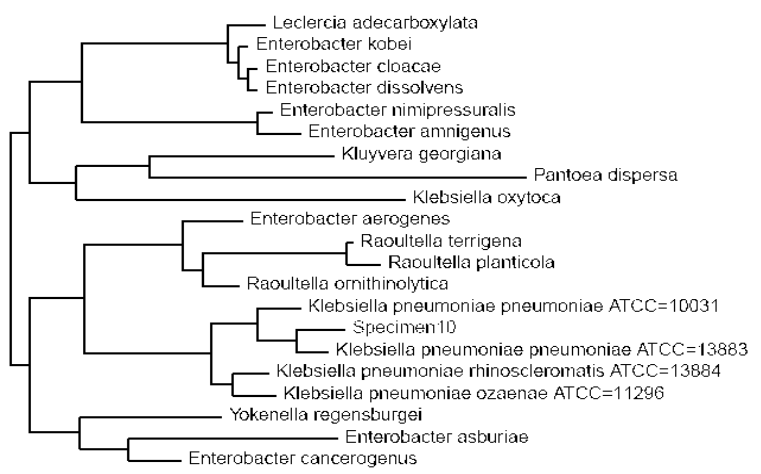

Fig. 13: Phylogenetic tree of Isolate $M$ constructed using neighbour joining method, constructed using neighbour joining method, showing $99.62 \%$ with $K$. pneumoniae

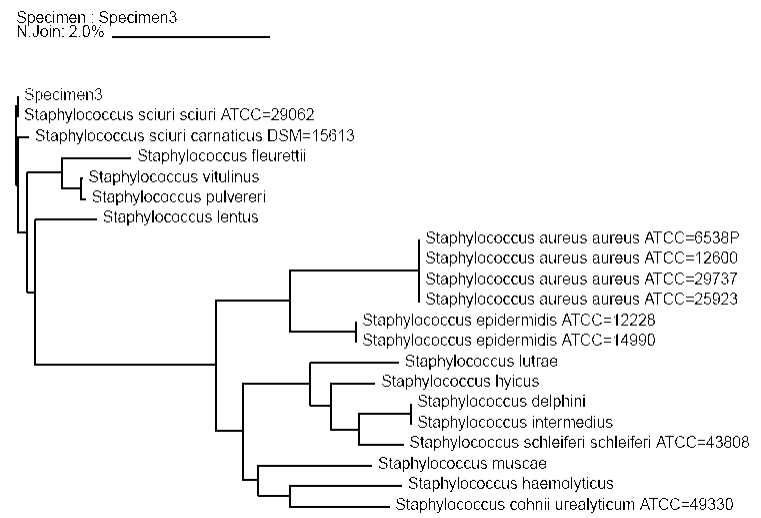

Fig. 14: Phylogenetic tree of Isolate $\mathrm{N}$ constructed using neighbour joining method, depicting $100 \%$ homology with S. sciuri

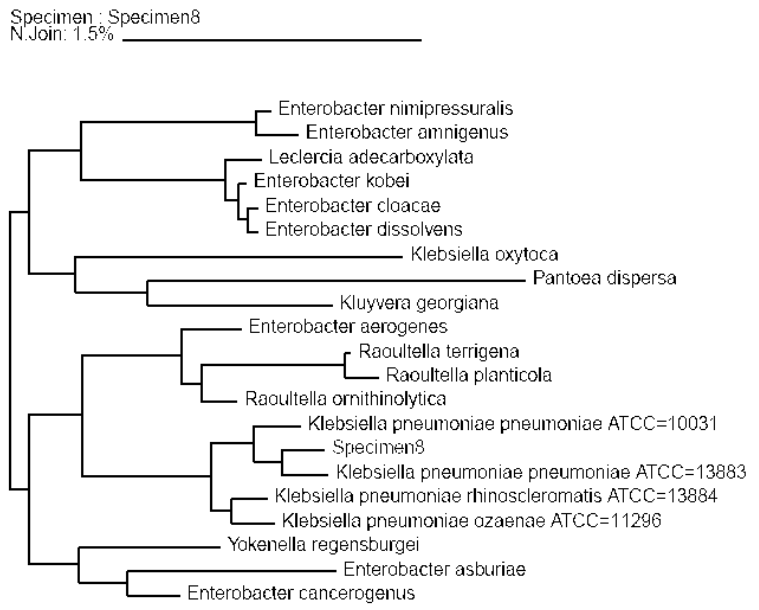

Fig. 15: Phylogenetic tree of Isolate $\mathrm{O}$ constructed using neighbour joining method, showing $99.57 \%$ similarity with $K$. pneumoniae

Thus, from the above study it is clear that molecular phylogeny along with biochemical analysis helps in precise identification of the microbe up to the strain level.

\section{ACKNOWLEDGEMENT}

The authors would like to acknowledge the financial support of BRNS, India as well as Department of Biotechnology, India. They would like to acknowledge the computational facility at the University developed through Department of Biotechnology, GOI grant. 


\section{REFERENCES}

1. http://patients. uptodate.com/topic. asp?file $=\mathrm{inf}$ immu/7633 (Accessed on 1.9.07).

2. Cattell, W.R. (Ed.), 1996. Infections of the Kidney and Urinary Tract. Oxford University Press. pp: 1-26.

3. http://www. health24. com /medical /Head 2 Toe/ 777-778-783,11914.asp (Accessed on 1.9.07).

4. http://kidney. niddk.nih.gov/ Kudiseases/ pubs/ utiadult/ (Accessed on 3.10.07).

5. http://www.textbookofbacteriology.net/e.coli.html (Accessed on 3.10.07). Todar's Online Textbook of Bacteriology. Pathogenic E. coli.

6. http://www. umm. edu/patiented /articles /what_ infectious _ agents _ that_cause _ urinary _ tract infections _000036_2.htm (Accessed on 3.10.07).

7. Pezzlo, M., 1988. Detection of Urinary Tract Infections by Rapid Methods. Clin. Microbiol. Rev., 1: 268-280.

8. Domann, E., G. Hong, C. Imizlioglu, J. Kuhle, C. Watzel, T. Hain, H. Hossain and T. Chakraborty, 2003. Culture Independent Identification of Pathogenic Bacteria and Polymicrobial Infections in the Genitourinary Tract of Tract of Renal Transplant Recipients. J. Clin. Microbiol., 41: 5500-5510.

9. http:// findarticles. com /p /articles /mi _ m2459 /is_n4_v24/ai_17450259 (Accessed on 1.9.07).

10. Garry, E., G. Ouattara, P. Williams and M. Pesta, 2006. Enumerating Chromogenic Agar Plates Using the Color QCOUNT Automated Colony Counter. IAFP, pp: 3-14. http: //www. aicompanies.com/ PDFs/ Articles/ Color \% 20 Qcount-IAFP\%202006-Booklet.pdf (accessed on 12.10.07).
11. www.sigmaaldrich. com/fluka/ product $\% 20$ information\% 20 sheet/16636 _ data - sheet _ $115 \mathrm{~kb}$. pdf (accessed on 30.6.07).

12. http://www. emedicine. com/ med/ topic 3089. htm (Accessed on 4.10.07).

13. Acharya, V.N. and S.K. Jadav, 1980. Urinary Tract Infection: Current Status. J. Postgraduate Medicine, 26: 95-98.

14. Manges, A.R., J.R. Johnson, B. Foxman, T.T. O'Bryan, K.E. Fullerton and L.W. Riley, 2001. Widespread Distribution of Urinary Tract Infections Caused by a Multidrug-Resistant Escherichia Coli Clonal Group. The New England J. Medicine, 345: 1007-1013.

15. Zhanel, G.G., N.M. Laing, K.A. Nichol, L.P. Palatnick, A. Noreddin, T. Hisanaga, J.L. Johnson, the NAVRESS Group and D.J. Hoban, 2003. Antibiotic Activity against Urinary Tract Infection (UTI) Isolates of Vancomycin-Resistant Enterococci (VRE): Results from the 2002 North American Vancomycin Resistant Enterococci Susceptibility Study (NAVRESS). J. Antimicrobial Chemotherapy, 52: 382-388. (Group), The New England J. Medicine, 345: 1007-1013.

16. Gales, A.C., R.N. Jones, K.A. Gordon, H.S. Sader, W.W. Wilke, M.L. Beach, M.A. Pfaller, G.V. Doern and the SENTRY Study Group (Latin America), 2000. Activity and Spectrum of 22 Antimicrobial Agents Tested against Urinary Tract Infection Pathogens in Hospitalized Patients in Latin America: Report from the Second Year of the SENTRY Antimicrobial Surveillance Program (1998). J. Antimicrobial Chemotherapy, 45: 295-303. 